\title{
Alterações hematológicas em pacientes acometidos pela COVID-19
}

\author{
Hematological changes in patients affected by COVID-19 \\ Cambios hematológicos en pacientes afectados por COVID-19
}

Recebido: 30/11/2021 | Revisado: 08/12/2021 | Aceito: 14/12/2021 | Publicado: 22/12/2021

Francisco Alex da Rocha Coelho

ORCID: https://orcid.org/0000-0002-3308-375X

Universidade Federal do Delta do Parnaíba, Brasil

E-mail: alex123rocha@hotmail.com

Rubens Renato de Sousa Carmo

ORCID: https://orcid.org/0000-0001-7135-7531

Universidade Federal do Delta do Parnaíba, Brasil

E-mail: rubensrenatobezerra@gmail.com

Wesley Rodrigues da Silva

ORCID: https://orcid.org/0000-0002-2241-5843

Universidade Federal do Delta do Parnaíba, Brasil

E-mail: mr.wesleyrodrigues@gmail.com

João Paulo Araújo de Sousa

ORCID: https://orcid.org/0000-0002-9255-8735

Universidade Federal do Delta do Parnaíba, Brasil E-mail: cigajp@ hotmail.com

Ana Clara de Moura Guimarães

ORCID: https://orcid.org/0000-0003-4328-3250

Universidade Federal do Delta do Parnaíba, Brasil

E-mail: anacmg29@yahoo.com.br

Letícia da Silva Queiroz

ORCID: https://orcid.org/0000-0002-2595-034X

Universidade Federal do Delta do Parnaíba, Brasil

E-mail: ldasilvaqueiroz330@gmail.com

Hélio Mateus Silva Nascimento

ORCID: https://orcid.org/0000-0003-1551-8139

Universidade Federal do Delta do Parnaíba, Brasil

E-mail: helio_mateus_@hotmail.com

Thais Amanda de Lima Nunes

ORCID: https://orcid.org/0000-0002-1924-4828

Universidade Federal do Delta do Parnaíba, Brasil

E-mail: amandalima-@hotmail.com.br

Vanessa dos Santos Brito

ORCID: https://orcid.org/0000-0001-6012-189X

Universidade Federal do Delta do Parnaíba, Brasil

E-mail: vnsx.biomed@gmail.com

John Arlley Sousa Pinho de Lira

ORCID https://orcid.org/0000-0001-6565-6260

Universidade Federal do Delta do Parnaíba, Brasi

E-mail: arlley_pinho@hotmail.com

Paulo Roberto Carneiro Gomes

ORCID: https://orcid.org/0000-0002-2911-7785

Universidade Federal do Delta do Parnaíba, Brasil

E-mail: paulo.c.gomes1@outlook.com

Even Herlany Pereira Alves

ORCID: https://orcid.org/0000-0001-7566-1282

Universidade Federal do Delta do Parnaíba, Brasi

E-mail: evenherlany@ufpi.edu.br

Julyanne Maria Saraiva de Sousa

ORCID: https://orcid.org/0000-0001-9041-0860

Universidade Federal do Delta do Parnaíba, Brasil

E-mail: jully.yanne@gmail.com

Airton Lucas Sousa dos Santos

ORCID: https://orcid.org/0000-0003-1975-9570

Universidade Federal do Delta do Parnaíba, Brasil

E-mail: airtonlucas@ufpi.edu.br

Paloma Maria de Sousa Araujo

ORCID: https://orcid.org/0000-0001-6185-8172

Universidade Federal do Delta do Parnaíba, Brasil

E-mail: psmaria1@ufpi.edu.br 


\begin{abstract}
Resumo
Este trabalho teve como foco avaliar as principais alterações provocadas no sistema sanguíneo em pacientes com COVID-19. Trata-se de uma revisão sistemática da literatura a partir de buscas nos bancos de dados PubMed e ScienceDirect, com a utilização de descritores específicos. Foram incluídos apenas estudos em suas versões completas, sem restrição de idiomas e publicados no recorte temporal de dezembro de 2019 a novembro de 2020 . O SARS-CoV-2 pode estar relacionado a diversas alterações no variados órgãos e sistemas, inclusive no sistema hematopoiético. As várias alterações hematológicas ocasionadas pela infecção decorrente do novo coronavírus se fazem de suma importância frente ao problema de saúde atual tendo em vista que tais alterações podem estar diretamente relacionadas ao mau prognóstico e até mesmo o óbito de muitos pacientes. O novo coronavírus é o sétimo da família a ser descrito associado à doença em seres humanos, tendo grande facilidade de transmissão de interpessoal e, portanto, pode se espalhar rapidamente. Quanto à sintomatologia, a COVID-19 é caracterizada por ser uma infecção com amplo espectro sintomatológico, podendo variar de um quadro totalmente assintomático a condições clínicas graves e com sintomas sistêmicos e em múltiplos órgãos. Diversas são as alterações no tecido hematopoiético, tendo destaque os distúrbios na cascata de coagulação, como incidência de eventos troboembólicos, elevação Tempo de Protrombina e o Tempo de Tromboplastina Parcial Ativada, seguido por uma contagem diminuída de plaquetas, aumento exacerbado de D-dímero, além da baixa atividade de coagulação de fibrinogênio.
\end{abstract}

Palavras-chave: Sistema hematopoiético; SARS-CoV-2; COVID-19.

\begin{abstract}
This work focused on evaluating the main changes caused in the blood system in patients with COVID-19. This is a systematic literature review based on searches in the PubMed and Science Direct databases, using specific descriptors. Only studies in their full versions, with no language restrictions and published in the time frame from December 2019 to November 2020, were included. SARS-CoV-2 may be related to several alterations in various organs and systems, including the hematopoietic system. The various hematological alterations caused by the infection resulting from the new coronavirus are of paramount importance given the current health problem, considering that such alterations may be directly related to the poor prognosis and even the death of many patients. The SARS-CoV-2 is the seventh in the family to be described as being associated with the disease in humans, having great ease of interpersonal transmission and, therefore, it can spread quickly. As for symptoms, COVID-19 is characterized as an infection with a broad symptomatological spectrum, which can range from a completely asymptomatic condition to severe clinical conditions with systemic symptoms and in multiple organs. There are several changes in hematopoietic tissue, with emphasis on disturbances in the coagulation cascade, such as the incidence of thromboembolic events, increase in Prothrombin Time and Activated Partial Thromboplastin Time, followed by a decreased platelet count, exacerbated increase in D-dimer, in addition to the low clotting activity of fibrinogen.
\end{abstract}

Keywords: Hematopoietic system; SARS-CoV-2; COVID-19.

\title{
Resumen
}

Este trabajo se centró en evaluar los principales cambios provocados en el sistema sanguíneo en pacientes con COVID-19. Es uma revisión sistemática de la literatura basada en búsquedas en las bases de datos PubMed y ScienceDirect, utilizando descriptores específicos. Solo se incluyeron estudios en su versión completa, sin restricciones de idioma y publicados en el período de tiempo de diciembre de 2019 a noviembre de 2020. El SARSCoV-2 puede estar relacionado con varias alteraciones en varios órganos y sistemas, incluido el sistema hematopoyético. Los diversos cambios hematológicos provocados por la infección resultante del nuevo coronavirus son de suma importancia ante el problema de salud actual, considerando que dichos cambios pueden estar directamente relacionados con un mal pronóstico e incluso con la muerte de muchos pacientes. El SARS-CoV-2 es el séptimo de la familia que se describe como asociado a la enfermedad en humanos, presenta una gran facilidad de transmisión interpersonal y, por tanto, puede propagarse rápidamente. En cuanto a los síntomas, el COVID-19 es una infección con amplio espectro sintomatológico, que puede ir desde una condición completamente asintomática hasta condiciones clínicas severas con síntomas sistémicos y en múltiples órganos. Hay varios cambios en el tejido hematopoyético, con énfasis en las alteraciones en la cascada de la coagulación, como la incidencia de eventos tromboembólicos, el aumento del tiempo de protrombina y el tiempo de tromboplastina parcial activada, seguidos de una disminución del recuento de plaquetas, un aumento exacerbado del dímero D, en además de la baja actividad de coagulación del fibrinógeno.

Palabras clave: Sistema hematopoyético; SARS-CoV-2; COVID-19.

\section{Introdução}

O novo coronavírus, identificado como SARS-CoV-2, é o sétimo da família a ser descrito associado à doença em seres humanos (Zhu et al., 2020). O SARS-CoV-2 é transmitido com eficiência de pessoa a pessoa e, portanto, pode se espalhar rapidamente. Na pandemia de COVID-19 em curso já foram notificados, até novembro de 2021, 262 milhões de casos 
de pessoas infectadas e 5,21 milhões de mortes em decorrência da doença (Our World in Data, 2021). A COVID-19 é uma doença aguda, possui tempo de incubação no corpo humano em torno de 4,1 a 7,0 dias e com média 5,2 dias, com intervalo de confiança de $95 \%$ para essa estimativa (Li, 2020a).

A infecção por COVID-19 pode ocorrer em três estágios possíveis: leve, moderado e grave. Pacientes mais jovens e sem comorbidades relevantes podem apresentar a doença em um estado leve ou moderado. Enquanto pacientes idosos, com comorbidades significativas, como diabetes e hipertensão, podem apresentar e predominar o estado grave da doença (SantosSánchez; Salas-Coronado, 2020; National Health Comission, 2020).

O SARS-CoV-2 pode estar associado às alterações em diversos órgãos e sistemas (Qi, et al., 2020). Alguns estudos mostraram que os sistemas respiratório, cardiovascular, digestivo e urinário são alvos da infecção por esse vírus. Além disso, variadas alterações hematológicas foram relatadas em diversos estudos e a relação de tais alterações ao diagnóstico e prognóstico da doença do coronavírus se faz relevante frente à pandemia atual (Mucha et al., 2020; Colling \& Kanthi, 2020; Iba et al., 2020; Liao et al., 2020; Vinayagam \& Sattu, 2020). Assim, este estudo teve como objetivo geral e específico avaliar o que se sabe, até o momento, sobre as principais alterações hematológicas presentes em pacientes acometidos pela COVID19.

\section{Metodologia}

O presente estudo trata-se de uma revisão sistemática da literatura, que segue metodologia descrita por LinaresEspinós e colaboradores (2018).

Foram feitas buscas nas bases de dados PubMed e ScienceDirect, com a utilização de descritores específicos: "hematological disorders", "blood paramenters", "COVID-19" e "red cell count" combinados entre si por meio de descritores booleanos, 1 AND e 2 OR. O processo de pesquisa ocorreu do dia 25 de outubro ao dia 16 de novembro de 2020.

Como critérios de inclusão: artigos em suas versões completas, sem restrição de idiomas, publicados no recorte temporal de dezembro de 2019 a novembro de 2020. Para a composição desta revisão foram considerados relatos de casos, artigos de revisão, artigos de pesquisa e estudos retrospectivos.

Estudos que não apresentavam informações relevantes em relação ao tema e que não obedeciam aos critérios de inclusão foram excluídos. Ademais, também foram excluídos os estudos repetidos.

Inicialmente, preconizou-se pela leitura dos trabalhos na íntegra para procura de potenciais estudos relevantes. Foram extraídas as seguintes informações contidas nos trabalhos incluídos: autores, ano de publicação, tipo de estudo e principais resultados. Após extrair as informações, os resultados foram comparados. (fonte: TNR 10 - justificado - espaço 1,5).

\section{Resultados}

A busca resultou em 597 estudos: 385 no PubMed e 212 na base de dados ScienceDirect. A princípio, 135 artigos foram excluídos por repetição. Restaram, portanto, 462. Destes, 444 artigos não atendiam aos critérios de inclusão e foram excluídos. Ao final, 18 estudos foram selecionados para compor esta revisão: 2 meta-análise, 10 artigos de revisão, 3 artigos de pesquisa e 3 estudos retrospectivos. 
Research, Society and Development, v. 10, n. 17, e117101724135, 2021

(CC BY 4.0) | ISSN 2525-3409 | DOI: http://dx.doi.org/10.33448/rsd-v10i17.24135

Figura 1. Fluxograma para a identificação, triagem, elegibilidade e análise dos estudos incluídos neste trabalho.

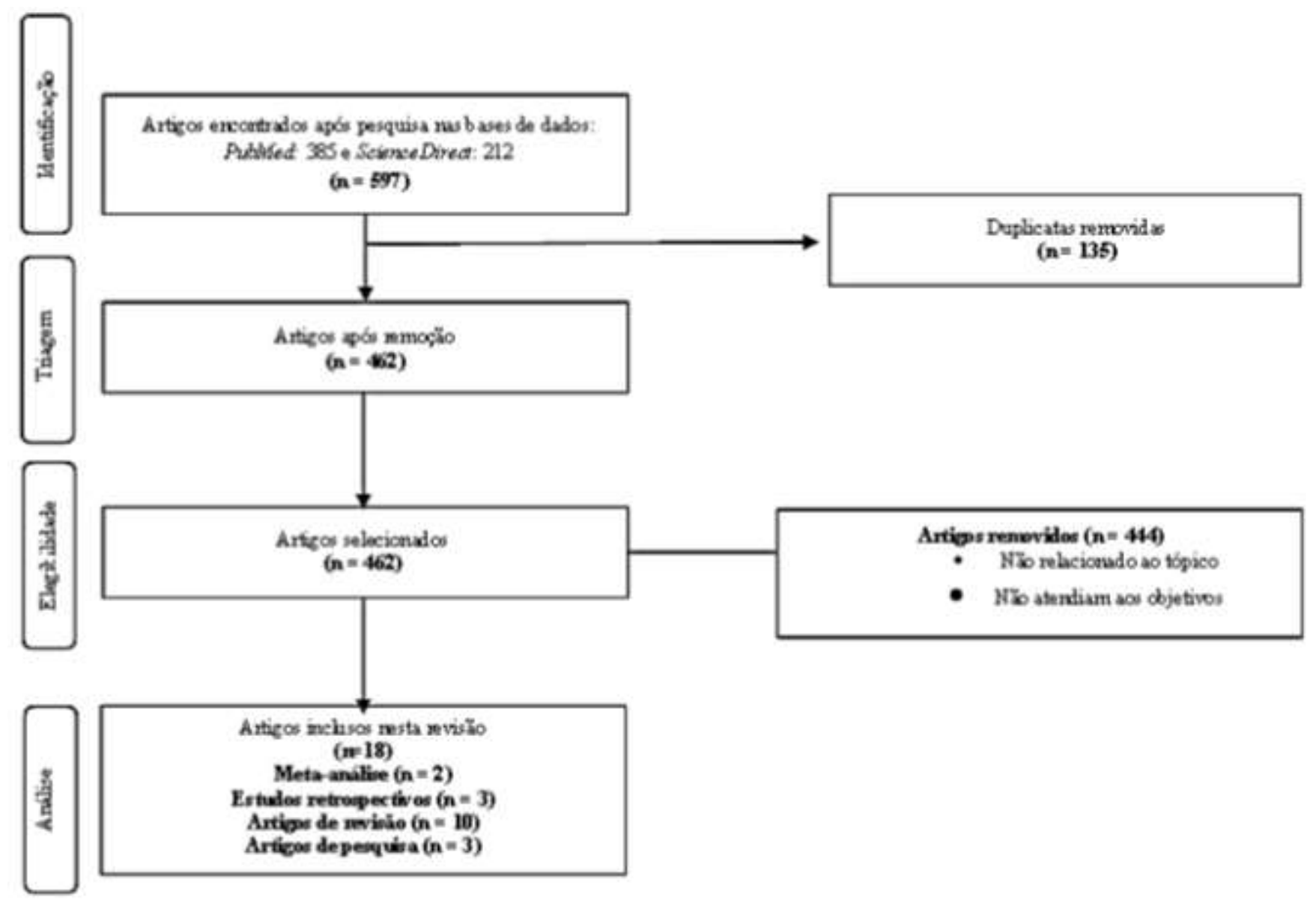

Fonte: Autores (2021). 
A Tabela abaixo mostra os principais resultados encontrados na literatura que relacionam alterações hematológicas em pacientes acometidos pela COVID-19, sendo detalhados por autor, ano de publicação, tipo de estudo e principais resultados encontrados em cada estudo em questão.

Tabela 1. Principais resultados da literatura acerca das alterações hematológicas em pacientes acometidos pela COVID-19.

\begin{tabular}{|c|c|c|}
\hline Autor(es) e ano de publicação & Tipo de estudo & Principais resultados \\
\hline (Frater et al., 2020) & Artigo de revisão & $\begin{array}{l}\text { O laboratório de hematologia fornece à equipe clínica uma série de marcadores prognósticos úteis para o diagnóstico e prognóstico dos pacientes. } \\
\text { Dentre os biomarcadores hematológicos de importância na infecção por COVID-19, destacam-se a linfopenia, leucocitose, neutrofilia } \\
\text { trombocitopenia. }\end{array}$ \\
\hline
\end{tabular}

(Giannis et al., 2020)

Artigo de revisão

As complicações trombóticas parecem surgir como uma questão importante nos pacientes com COVID-19. Os resultados mostraram que os pacientes infectados comumente desenvolvem trombocitopenia (36,2\%) e podem ter D-dímero elevado (46,4\%), embora essas taxas sejam ainda maiores em pacientes graves. Segundo dados, pacientes infectados pelo SARS-CoV-2 estão em risco de desenvolver CIVD. Níveis aumentados de D-dímero, produtos de degradação de fibrina e TP prolongados foram associados a mau prognóstico em pacientes com COVID-19.

\begin{abstract}
(Miesback \& Makris, 2020)
Estudo de coorte retrospectivo

A coagulopatia foi descrita em estudos que documentam alterações clínicas e laboratoriais em pacientes com COVID-19 em até 50\% daqueles com manifestações graves. O aumento no dímero- D foi a alteração mais significativa nos parâmetros de coagulação em pacientes com COVID-19 e ocorreu com mais frequência do que outros parâmetros de coagulação, como o TP ou TTPa. Além disso, os parâmetros de coagulação indicam uma tendência acentuada à trombose, uma vez que as alterações de outros parâmetros que indicam uma tendência ao sangramento, como plaquetas gravemente baixas ou níveis de fibrinogênio, estavam ausentes.
\end{abstract}

A linfopenia pode ser considerada um achado laboratorial cardinal, com potencial prognóstico. A proporção de neutrófilos/linfócitos e a proporção

(Terpos et al., 2020)

Artigo de revisão

de pico de plaquetas/linfócitos também podem ter valor prognóstico na determinação de casos graves. Durante o curso da doença, a avaliação longitudinal da dinâmica da contagem de linfócitos e índices inflamatórios, incluindo LDH, CRP e IL-6, pode ajudar a identificar casos com prognóstico sombrio e intervenção imediata para melhorar os resultados.

Foram incluídos 388 pacientes. Eventos tromboembólicos ocorreram em 28 correspondendo a uma taxa cumulativa de $21 \%$. Metade dos eventos o TEV foi confirmado em 16. A angiotomografia pulmonar (AFC) foi realizada em 30 pacientes, correspondendo a 7,7\% do total, e a embolia pulmonar foi confirmada em 10. A taxa de acidente vascular cerebral isquêmico e ACS / MI foi de 2,5\% e 1,1\%, respectivamente. A CID aberta esteve presente em 8 pacientes. 
Os parâmetros de TEG são consistentes com um estado de hipercoagulabilidade, conforme mostrado por valores diminuídos e valores aumentados do ângulo K e MA. A contagem de plaquetas estava normal ou aumentada, o tempo de protrombina e o tempo de tromboplastina parcial ativada estavam próximos (normal). O fibrinogênio foi aumentado e o D-dímero foi dramaticamente aumentado. A proteína C reativa foi aumentada. Fator VIII e fator de von Willebrand foram aumentados. A antitrombina diminuiu marginalmente e a proteína aumentou.

(Lippi et al.,2020) Meta-análise

Nove estudos com 1779 pacientes COVID-19, 399 com doença grave, foram incluídos na meta-análise. A análise combinada revelou que a contagem de plaquetas foi significativamente menor em pacientes com COVID-19 mais grave. Uma análise de subgrupo comparando pacientes por sobrevida, descobriu que uma contagem de plaquetas ainda mais baixa foi observada com mortalidade. Em quatro dos estudos que relatou dados sobre a taxa de trombocitopenia, uma baixa contagem de plaquetas foi associada a um risco cinco vezes maior de COVID-19 grave.

Parâmetros de coagulação anormais em 183 pacientes consecutivos com COVID-19. Os níveis basais de PT, dímero D e produtos de degradação da fibrina aumentaram significativamente em não sobreviventes em comparação com os sobreviventes. Além disso, 71,4\% dos pacientes com resultado fatal desenvolveram uma coagulopatia sistêmica progressiva ao longo da hospitalização, que era consistente DIC de acordo com os critérios da ISTH. Em estágios posteriores, a CID em não sobreviventes foi caracterizada por dímero D e consumo de fibrinogênio maciçamente elevados, um achado consistente com fibrinólise excessiva.

(Boccia et al., 2020)

Artigo de revisão

A COVID-19 é com frequência complicada pela presença de alguma coagulopatia, sendo a DIC presente na maioria dos pacientes falecidos. Os parâmetros convencionais de coagulação parecem estar significativamente alterados em pacientes com mau prognóstico. Uma ampla linha cruzada entre hemostasia coagulativa e inflamação, bem como a ativação da cascata de coagulação durante infecções virais, está bem estabelecida. Outra evidência importante que pode explicar os distúrbios da coagulação em COVID-19 é o aumento da formação de trombos em condições de hipóxia.

Implicações vasculares da COVID-19 podem resultar em proteínas de coagulação e biomarcadores circulantes, disfunções endoteliais e eritrocitárias e plaquetárias. De particular interesse são os vários biomarcadores de coagulação inflamatória circulantes envolvidos diretamente na coagulação, com foco específico em fibrina, dímero D, selectina P e VWF. Mudanças em seus níveis podem levar a um desequilíbrio entre fatores pró-coagulantes e anticoagulantes.

Em primeiro momento, comparou-se as covariáveis hematológicas na admissão entre sobreviventes e não sobreviventes. Indivíduos que morreram tiveram uma mediana de WBC mais alta, neutrófilos medianos mais elevados, linfócitos medianos inferiores e plaquetas medianas inferiores em comparação com os sobreviventes. Além disso, pode-se perceber que os valores basais e máximos do TP, TTPa e concentrações de dímero D foram significativamente maiores em indivíduos que morreram em comparação com os sobreviventes.

A análise da redução dinâmica na contagem de plaquetas pode ser útil no prognóstico de pacientes com COVID-19. Pacientes com doença grave e

(Zhang et al., 2020a)

Artigo de revisão desfechos fatais apresentam uma proporção diminuída de linfócitos/leucócitos em comparação com os pacientes não graves. Pacientes graves e/ou críticos tiveram contagem de plaquetas diminuída bem como distúrbios de coagulação. Um estudo retrospectivo semelhante realizado em pacientes com COVID-19 mostrou uma correlação de três vezes entre trombocitopenia e mortalidade em comparação com pacientes sem trombocitopenia

Em comparação com pacientes regulares, pacientes graves e criticamente enfermos tiveram contagem de linfócitos significativamente mais baixa, neutrófilos, IL-6, PCR, taxa de sedimentação de eritrócitos, ferritina e LDH. 
(Liao et al., 2020) Estudo de coorte retrospectivo

(Fathi \& Rezaei, 2020)

Artigo de revisão

De 466 pacientes internados, a incidência de trombocitopenia em pacientes com doença crítica foi significativamente maior do que naqueles com doença grave ou moderada. Os números de linfócitos e eosinófilos foram significativamente menores em pacientes com doença crítica do que aqueles com doença grave ou moderada, e o TP, dímero D e produtos de degradação da fibrina aumentaram significativamente com o aumento da gravidade da doença. Eventos trombóticos e hemorrágicos foram complicações comuns em pacientes que morreram.

Em estudos recentes, foi demonstrado que cerca de $85 \%$ dos pacientes gravemente enfermos de COVID-19 sofrem de linfopenia. Embora as células T pudessem estar inicialmente aumentadas no início da COVID-19, esses pacientes tendiam a ter baixa contagem de linfócitos. Portanto, os indivíduos que morreram de COVID-19 demonstraram ter níveis de linfócitos expressivamente mais baixos do que os sobreviventes.

(Pourbagheri-Sigaroodi, 2020)

Artigo de revisão

A contagem total de leucócitos varia entre os pacientes, o que pode refletir a predominância de linfopenia ou neutrofilia. A diminuição dos linfócitos acompanhada de trombocitopenia leve estão entre os achados anormais mais comuns que chamam a atenção no hemograma completo de pacientes com COVID-19. Também foi relatado que alguns pacientes com COVID-19 aumentaram o TP juntamente com o tempo prolongado de TTPa. Somando-se a essas anormalidades, dímeros D elevados apoiam ainda mais a ocorrência de coagulopatia. A COVID-19 não faz exceção a essa regra, segundo a qual a VHS, PCR estão aumentados.

Leucopenia ou contagem total de leucócitos normal, linfopenia e trombocitopenia leve a moderada são comumente observadas. Linfopenia e (Kasinathan \& Sathar, 2020) Artigo de revisão trombocitopenia parecem predizer a gravidade da doença para COVID-19.

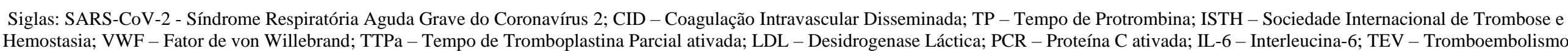
venoso; VHS - Velocidade de hemossedimentação. Fonte: Autores (2021). 


\section{Discussão}

A importância desta revisão baseia-se na demonstração, através de dados encontrados na literatura, das implicações causadas pela COVID-19 sobre os parâmetros hematológicos, as consequências destas implicações e, dessa maneira, incitar pesquisas sobre a temática aqui abordada. A doença infecciosa causada pelo novo coronavírus se expandiu rapidamente pelo mundo causando várias mortes, afetando o sistema de saúde e a economia global (Chen, 2020; Bodbey \& Ray, 2020). De acordo com os resultados encontrados, a literatura apresenta evidências de que o patógeno causador dessa doença afeta diversos sistemas e tecidos do organismo humano, dentre eles o sistema hematopoético (Langer et al., 2020; Boccia et al., 2020; Zhang et al., 2020a; Giannis et al., 2020; Miesback \& Makris, 2020).

$\mathrm{Na}$ infecção pelo SARS-CoV-2, grande parte dos pacientes apresentam apenas sintomas leves. No entanto, uma característica da COVID-19 é que uma proporção de pacientes manifestar complicações graves em um pequeno espaço de tempo após a infecção, como síndrome respiratória aguda grave (SDRA) ou coagulação intravascular disseminada (CIVD), sepse seguida por falência de órgãos e morte (Miesback \& Makris, 2020; Huang et al., 2020; Yang et al., 2020).

Os distúrbios na cascata de coagulação são encontrados com relativa frequência entre os pacientes com COVID-19, principalmente entre os pacientes com doença grave (Terpos et al., 2020; Deng et al., 2020; Zhou et al., 2020). Uma metaanálise de nove estudos sugeriu que a trombocitopenia está significativamente associada à gravidade da doença COVID-19, com uma heterogeneidade muito alta entre os estudos, sendo uma queda mais considerável na contagem de plaquetas observada especialmente em não sobreviventes (Terpos, et al., 2020; Lippi et al., 2020).

Além disso, as complicações tromboembólicas podem figurar uma parte constituinte do quadro clínico da COVID-19 e já estão presentes no momento da admissão hospitalar inicial. No entanto, tendo em vista o pequeno número de exames de imagem específicos realizados, sua incidência pode ter sido altamente subestimada. Neste mesmo estudo, mostrou-se que a maioria das complicações trombóticas era venosa e representada principalmente por embolia pulmonar (EP) de forma isolada (Lodigiani et al., 2020).

Em soma, diversos estudos demonstram, evidentemente, a relação da COVID-19 com outros parâmetros hematológicos, sendo o resultado destes importantes para o prognóstico e melhor decisão acerca de qual medida terapêutica ou profilática o paciente possa ser submetido. Ademais, tais resultados podem estar, frequentemente, associados ao aumento de mortalidade. Dentre os resultados presentes nos vários estudos, destacam-se o Tempo de Protrombina (TP) e o Tempo de Tromboplastina Parcial Ativada (TTPa) aumentados, seguido por uma contagem diminuída de plaquetas, aumento exacerbado de D-dímero, além da baixa atividade de coagulação de fibrinogênio. Estes resultados estão diretamente relacionados a um estado de hipercoagulabilidade, sendo sinais patognomônicos da coagulação intravascular disseminada (CIVD), aumentado, dessa forma, a propensão para o desenvolvimento de outros distúrbios da coagulação como que podem ser fatais, como EP e trombose venosa profunda (Panigada et al., 2020; Liao et al., 2020; Li et al., 2020b; Pourbagheri-Sigarood, 2020; Yuan et al., 2020; Grobler et al., 2020).

Outrossim, em outros estudos a linfopenia foi um achado comum em pacientes com infecção por COVID-19 e acredita-se que represente uma resposta imunológica defeituosa ao vírus. A leucocitose, independentemente de representar uma neutrofilia, linfocitose ou ambas, é observada em uma minoria de pacientes infectados por COVID-19 e parece anunciar infecção bacteriana ou superinfecção. Os dados sobre neutrofilia são incompletos e não foram amplamente abordados na literatura. Os dados disponíveis sugerem que a neutrofilia é uma expressão da tempestade de citocinas e do estado hiperinflamatório que têm um papel patogenético importante no COVID-19 e em infecções relacionadas, como a SARS-CoV2 (Father et al., 2020; Fathi \& Rezaei, 2020; Liao et al., 2020; Zhang et al., 2020a; Terpos et al., 2020; Kasinathan \& Sathar, 2020). 


\section{Conclusão}

Portanto, o presente trabalho teve como foco principal avaliar as possíveis alterações decorrentes da infecção pelo novo coronavírus sobre o sistema hematopoiético. Assim, foi possível observar que as diversas alterações no tecido sanguíneo, principalmente na forma grave da COVID-19, podem estar intimamente relacionadas ao mau prognóstico e, até mesmo, ser a causa mortis de muitos pacientes. Desta forma, faz-se necessário a execução de mais estudos para o melhor entendimento dos reais mecanismos fisiopatológicos utilizados pelo SARS-CoV-2 e, consequentemente, o desenvolvimento da terapêutica mais adequada.

\section{Referências}

Boccia, M., Aronne, L., Celia, B., Mazzeo, G., Ceparano, M., D'Agnano, V., ... \& Perrotta, F. (2020). COVID-19 and coagulative axis: review of emerging aspects in a novel disease. Monaldi Archives for Chest Disease, 90(2).

Chen, N., Zhou, M., Dong, X., Qu, J., Gong, F., Han, Y., ... \& Zhang, L. (2020). Epidemiological and clinical characteristics of 99 cases of 2019 novel coronavirus pneumonia in Wuhan, China: a descriptive study. The lancet, 395(10223), 507-513.

Colling, M. E., \& Kanthi, Y. (2020). <? covid19?> COVID-19-associated coagulopathy: An exploration of mechanisms. Vascular Medicine, 25(5), 471-478.

Deng, Y., Liu, W., Liu, K., Fang, Y. Y., Shang, J., Zhou, L., ... \& Liu, H. G. (2020). Clinical characteristics of fatal and recovered cases of coronavirus disease 2019 in Wuhan, China: a retrospective study. Chinese medical journal, 133(11), 1261.

Fathi, N., \& Rezaei, N. (2020). Lymphopenia in COVID-19: Therapeutic opportunities. Cell biology international, 44(9), 1792-1797.

Frater, J. L., Zini, G., d'Onofrio, G., \& Rogers, H. J. (2020). COVID-19 and the clinical hematology laboratory. International journal of laboratory hematology, 42, 11-18.

Giannis, D., Ziogas, I. A., \& Gianni, P. (2020). Coagulation disorders in coronavirus infected patients: COVID-19, SARS-CoV-1, MERS-CoV and lessons from the past. Journal of Clinical Virology, 127, 104362.

Grobler, C., Maphumulo, S. C., Grobbelaar, L. M., Bredenkamp, J. C., Laubscher, G. J., Lourens, P. J., ... \& Pretorius, E. (2020). Covid-19: The rollercoaster of fibrin (ogen), d-dimer, von willebrand factor, p-selectin and their interactions with endothelial cells, platelets and erythrocytes. International journal of molecular sciences, 21(14), 5168

Huang, C., Wang, Y., Li, X., Ren, L., Zhao, J., Hu, Y., ... \& Cao, B. (2020). Clinical features of patients infected with 2019 novel coronavirus in Wuhan, China. The lancet, 395(10223), 497-506.

Iba, T., Levy, J. H., Levi, M., Connors, J. M., \& Thachil, J. (2020). Coagulopathy of coronavirus disease 2019. Critical care medicine.

Kasinathan, G., Sathar, J. Haematological manifestations, mechanisms of thrombosis and anti-coagulation in COVID-19 disease: a review. Annals of Medicine and Surgery, 2020.

Langer, F., Kluge, S., Klamroth, R., \& Oldenburg, J. (2020). Coagulopathy in COVID-19 and its implication for safe and efficacious thromboprophylaxis. Hämostaseologie, 40(03), 264-269.

Li, Q., Guan, X., Wu, P., Wang, X., Zhou, L., Tong, Y., ... \& Feng, Z. (2020a). Early transmission dynamics in Wuhan, China, of novel coronavirus-infected pneumonia. New England journal of medicine.

Li, Q., Cao, Y., Chen, L., Wu, D., Yu, J., Wang, H., ... \& Hu, Y. (2020b). Hematological features of persons with COVID-19. Leukemia, 34(8), $2163-2172$.

Liao, D., Zhou, F., Luo, L., Xu, M., Wang, H., Xia, J., ... \& Hu, Y. (2020). Haematological characteristics and risk factors in the classification and prognosis evaluation of COVID-19: a retrospective cohort study. The Lancet Haematology, 7(9), e671-e678.

Linares-Espinós, E., Hernández, V., Domínguez-Escrig, J. L., Fernández-Pello, S., Hevia, V., Mayor, J.,... \& Ribal, M. J. (2018). Methodology of a systematic review. Metodología de una revisión sistemática. Actas urologicas espanolas, 42(8), 499-506.

Lippi, G., Plebani, M., \& Henry, B. M. (2020). Thrombocytopenia is associated with severe coronavirus disease 2019 (COVID-19) infections: a meta-analysis. Clinica chimica acta, 506, 145-148.

Lodigiani, C., Iapichino, G., Carenzo, L., Cecconi, M., Ferrazzi, P., Sebastian, T., ... \& Force, T. (2020). Venous and arterial thromboembolic complications in COVID-19 patients admitted to an academic hospital in Milan, Italy. Thrombosis research, 191, 9-14.

Ludwig, S., \& Zarbock, A. (2020). Coronaviruses and SARS-CoV-2: a brief overview. Anesthesia and analgesia.

Mucha, S. R. et al. (2020). Coagulopathy in COVID-19: Manifestations and management. Cleveland Clinic journal of medicine, 87(8), 461.

National Health Commission. (2020). Diagnosis and treatment protocol for novel coronavirus pneumonia (Trial Version 7). Chinese Medical Journal (Engl), 133(9), 1087-1095. 
Research, Society and Development, v. 10, n. 17, e117101724135, 2021 (CC BY 4.0) | ISSN 2525-3409 | DOI: http://dx.doi.org/10.33448/rsd-v10i17.24135

Our World in Data. Coronavirus Pandemic (COVID-19) - the data. https://ourworldindata.org/coronavirus-data.

Pang, J., Wang, M. X., Ang, I. Y. H., Tan, S. H. X., Lewis, R. F., Chen, J. I. P., ... \& Hsu, L. Y. (2020). Potential rapid diagnostics, vaccine and therapeutics for 2019 novel coronavirus (2019-nCoV): a systematic review. Journal of clinical medicine, 9(3), 623.

Panigada, M., Bottino, N., Tagliabue, P., Grasselli, G., Novembrino, C., Chantarangkul, V., ... \& Tripodi, A. (2020). Hypercoagulability of COVID-19 patients in intensive care unit: a report of thromboelastography findings and other parameters of hemostasis. Journal of Thrombosis and Haemostasis, 18(7), 17381742 .

Pourbagheri-Sigaroodi, A., Bashash, D., Fateh, F., \& Abolghasemi, H. (2020). Laboratory findings in COVID-19 diagnosis and prognosis. Clinica Chimica Acta; International Journal of Clinical Chemistry, 510, 475.

Qi, J., Zhou, Y., Hua, J., Zhang, L., Bian, J., Liu, B., ... \& Jin, S. (2021). The scRNA-seq expression profiling of the receptor ACE2 and the cellular protease TMPRSS2 reveals human organs susceptible to SARS-CoV-2 infection. International Journal of Environmental Research and Public Health, $18(1), 284$.

Santos-Sánchez, N. F., \& Salas-Coronado, R. (2020). Origin, structural characteristics, prevention measures, diagnosis and potential drugs to prevent and COVID-19. Medwave, 20(8).

Terpos, E., Ntanasis-Stathopoulos, I., Elalamy, I., Kastritis, E., Sergentanis, T. N., Politou, M., ... \& Dimopoulos, M. A. (2020). Hematological findings and complications of COVID-19. American journal of hematology, 95(7), 834-847.

Vinayagam, S., \& Sattu, K. (2020). SARS-CoV-2 and coagulation disorders in different organs. Life Sciences, 118431.

Yang, X., Yu, Y., Xu, J., Shu, H., Liu, H., Wu, Y., ... \& Shang, Y. (2020). Clinical course and outcomes of critically ill patients with SARS-CoV-2 pneumonia in Wuhan, China: a single-centered, retrospective, observational study. The Lancet Respiratory Medicine, 8(5), 475-481.

Yuan, X., Huang, W., Ye, B., Chen, C., Huang, R., Wu, F., ... \& Hu, J. (2020). Changes of hematological and immunological parameters in COVID-19 patients. International journal of hematology, 112(4), 553-559.

Zhang, Y., Zeng, X., Jiao, Y., Li, Z., Liu, Q., Ye, J., \& Yang, M. (2020a). Mechanisms involved in the development of thrombocytopenia in patients with COVID-19. Thrombosis research, 193, 110-115.

Zhang, Z. L., Hou, Y. L., Li, D. T., \& Li, F. Z. (2020b). Laboratory findings of COVID-19: a systematic review and meta-analysis. Scandinavian journal of clinical and laboratory investigation, 80(6), 441-447.

Zhou, F., Yu, T., Du, R., Fan, G., Liu, Y., Liu, Z., ... \& Cao, B. (2020). Clinical course and risk factors for mortality of adult inpatients with COVID-19 in Wuhan, China: a retrospective cohort study. The lancet, 395(10229), 1054-1062.

Zhu, J., Ji, P., Pang, J., Zhong, Z., Li, H., He, C., ... \& Zhao, C. (2020). Clinical characteristics of 3062 COVID-19 patients: a meta-analysis. Journal of medical virology, 92(10), 1902-1914. 